\title{
Multi-Wavelength Narrow Linewidth Fiber Laser Based on Distributed Feedback Fiber Lasers
}

\author{
Jingsheng $\mathrm{LV}^{1}$, Haifeng $\mathrm{QI}^{1^{*}}$, Zhiqiang $\mathrm{SONG}^{1}$, Jian $\mathrm{GUO}^{1}$, Jiasheng $\mathrm{NI}^{1}$, \\ Chang WANG ${ }^{1}$, and Gangding PENG ${ }^{1,2}$

\begin{abstract}
${ }^{1}$ Shandong Key Laboratory of Optical Fiber Sensing Technologies, Laser Institute of Shandong Academy of Sciences, Jinan, 250014, China

${ }^{2}$ School of Electrical Engineering \& Telecommunications, The University of New South Wales, NSW, 2052, Australia

*Corresponding author: Haifeng QI E-mail: qihf@sdlaser.cn
\end{abstract}

\begin{abstract}
A narrow linewidth laser configuration based on distributed feedback fiber lasers (DFB-FL) with eight wavelengths in the international telecommunication union (ITU) grid is presented and realized. In this laser configuration, eight phase-shifted gratings in series are bidirectionally pumped by two $980-\mathrm{nm}$ laser diodes (LDs). The final laser output with over 10-mW power for each wavelength can be obtained, and the maximum power difference within eight wavelengths is $1.2 \mathrm{~dB}$. The laser configuration with multiple wavelengths and uniform power outputs can be very useful in large scaled optical fiber hydrophone fields.
\end{abstract}

Keywords: Multi-wavelength fiber laser; distributed feedback fiber laser; narrow linewidth fiber laser

Citation: Jingsheng LV, Haifeng QI, Zhiqiang SONG, Jian GUO, Jiasheng NI, Chang WANG, et al., "Multi-Wavelength Narrow Linewidth Fiber Laser Based on Distributed Feedback Fiber Lasers," Photonic Sensors, 2016, 6(3): 256-260.

\section{Introduction}

Narrow linewidth fiber lasers or semiconductor lasers with fiber output connectors are widely used as laser sources in high sensitive optical fiber sensing fields, such as submarine hydroacoustic detecting and distributed acoustic sensing [1, 2]. Currently, the practical optical fiber hydrophone system is based on the optical fiber interferometer technology. A multi-wavelength narrow linewidth laser or a laser array composed of multiple single-wavelength narrow linewidth lasers, where the multiple wavelengths should be in the international telecommunication union (ITU) grid, is needed for the large scaled hydrophone array [3]. However, the laser array composed of multiple single-wavelength laser modules is always cumbersome and complicated in the configuration. Hence, a laser with the multi-wavelength output is attractive for its compact structure and usability. In recent years, the narrow linewidth fiber laser with the compact structure has been realized in distributed feedback fiber laser (DFB-FL) where a short phase shifted fiber grating in erbium doped fiber as the distributed feedback laser cavity assures the robust single longitudinal mode operation [4-7]. This distributed feedback fiber laser can be used as a sensing element in the high sensitive acoustic detecting field and has been extensively studied previously [8,9]. Meanwhile, it can be used as a seed laser of the high performance laser source for its excellent single frequency narrow linewidth

Received: 11 April 2016 / Revised: 6 June 2016

(C) The Author(s) 2016. This article is published with open access at Springerlink.com

DOI: $10.1007 / \mathrm{s} 13320-016-0330-\mathrm{z}$

Article type: Regular 
characteristic and simple structure [10].

In this paper, based on DFB-FLs, a narrow linewidth laser configuration with eight wavelengths in the $\mathrm{C}$ band is presented and realized. The eight wavelengths are in the ITU grid with a spectral separation of $1.6 \mathrm{~nm}(200 \mathrm{GHz})$. In the laser configuration, eight phase-shifted gratings of eight different wavelengths in the ITU grid are pumped bidirectionally by two $980-\mathrm{nm}$ LDs in series. The final laser output with over $10-\mathrm{mW}$ power for each wavelength can be obtained after the subsequent amplification by an erbium doped fiber amplifier (EDFA) structure. The maximum power difference within the eight wavelengths is $1.2 \mathrm{~dB}$. The laser configuration with multiple wavelengths and uniform power outputs should be a practical laser source in the large scaled optical fiber hydrophone system.

\section{Experiments}

The schematic diagram of the eight-wavelength narrow linewidth fiber laser configuration based on DFB-FLs is shown in Fig. 1. The eight phase-shifted gratings on the erbium doped fiber have the same structure parameters except the central wavelengths. The eight wavelengths are $1553.292 \mathrm{~nm}$, $1551.788 \mathrm{~nm}, 1550.010 \mathrm{~nm}, 1548.475 \mathrm{~nm}$, $1546.883 \mathrm{~nm}, 1545.301 \mathrm{~nm}, 1543.658 \mathrm{~nm}$, and $1542.095 \mathrm{~nm}$, respectively, which are just in accord with C30, C32, C34, C36, C38, C40, C42, and C44 ITU channels. The grating length abbreviated as $L_{g}$ is $40 \mathrm{~mm}$, and a discrete $\pi$ phase shift is located at $0.4 * L_{g}$ to assure the almost unidirectional output with an over 20-dB backward-to-forward output

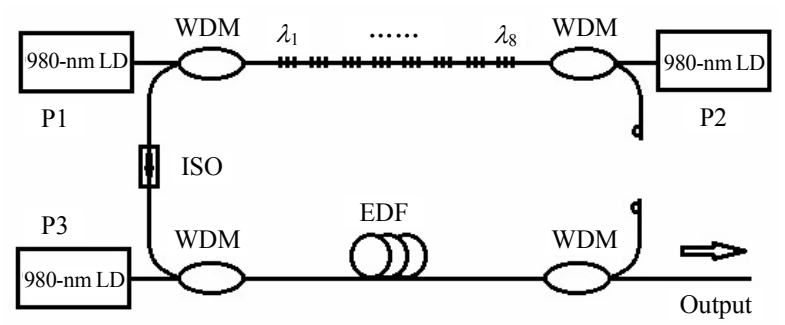

Fig. 1 Schematic diagram of the presented eight-wavelength laser configuration. power ratio. The detailed fabrication procedure has been reported particularly in previous publications $[6,7]$. For each phase-shifted grating, a 4-cm-long phase-shift grating is written on a 5-cm-long photosensitive erbium doped fiber (Nufern, the peak absorption at $1530 \mathrm{~nm}$ is $8 \mathrm{~dB} / \mathrm{m}$ ) which is spliced to the passive matching pigtail fiber (Nufern $980 \mathrm{Hp}$ ) at both ends. The grating is written by scanning 244-nm frequency-doubled harmonic Argon ion continuous wave laser across the phase mask and fiber. A polarization dependent grating is generated by the vertically polarized ultraviolet laser. In the fabrication process, the phase shift is introduced by a simple relative movement of the phase mask to the fiber during the beam scanning. The movement of the phase mask for an accurate $\pi$ phase-shift is a quarter of the period of the phase mask which is mounted on a piezoelectric transducer (PZT) actuator (PI, P-752.11c) with a nanometer resolution. Different from the shield method and post processing method to get a phase-shifted grating, by using this technique, the generated phase shift is only dependent on the relative displacement of the phase mask without relation to the ultraviolet laser induced index change in the grating. The eight phase-shifted gratings served as eight laser cavities are spliced in series in the same direction. This serial cavity array is pumped from both directions. After adjusting P1 and P2 properly, where P1 is $50 \mathrm{~mW}$ and $\mathrm{P} 2$ is $260 \mathrm{mw}$, the output from the isolator (ISO) has a relatively uniform power distribution where the maximum power difference within the eight wavelengths is $1.4 \mathrm{~dB}$ (Fig. 2, solid line). The total output power is $1.5 \mathrm{~mW}$ measured with EXFO FPM-600 power meter. To get a high and practical power, the laser output from the ISO is subsequently amplified by a forward-pumped EDFA structure. A 500-mW 980-nm LD and a 4.5-m-long erbium doped fiber (EDF) (Er-doped concentration: $4000 \mathrm{ppm}$ ) with an absorption of $7 \mathrm{~dB} / \mathrm{m}$ at $980 \mathrm{~nm}$ are applied here. The final output power is $120 \mathrm{~mW}$, and the maximum power difference within the eight 
wavelengths is $1.2 \mathrm{~dB}$ (Fig. 2, dashed line). The laser spectrum in the 50-nm range measured with an optical spectrum analyzer (YOKOGAWA AQ6370) is shown in Fig. 3.

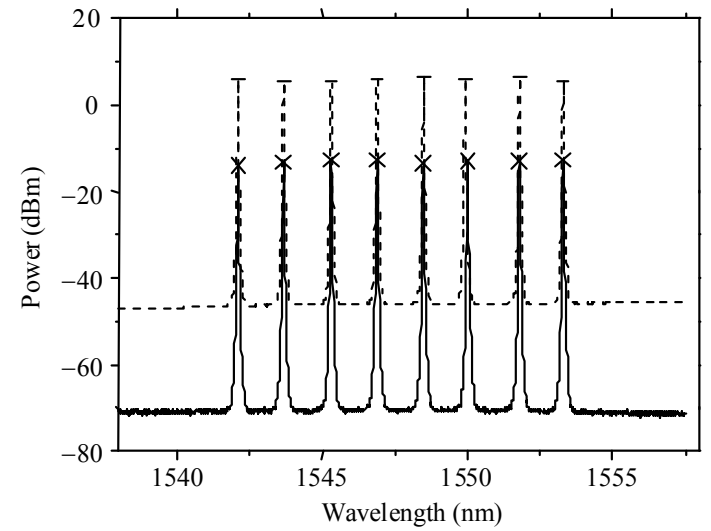

Fig. 2 Final eight-wavelength laser output before (solid line) and after (dashed line) amplification.

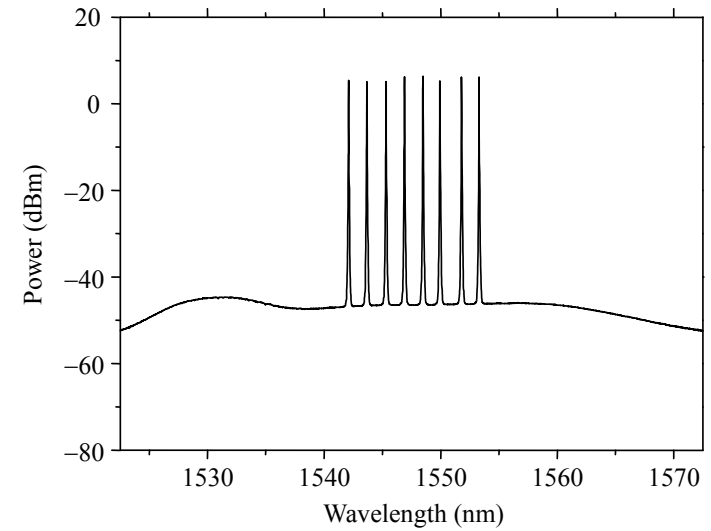

Fig. 3 Final eight-wavelength laser output measured in the 50-nm spectrum range.

For more detailed characteristic analysis, the laser in C34 channel is filtered out by a dense wavelength division multiplexing (DWDM) filter, and its output power is tested to be $14 \mathrm{~mW}$. Its linewidth and relative intensity noise (RIN) are tested to be $1.5 \mathrm{kHz}$ and $-100 \mathrm{~dB} / \mathrm{Hz}$ at the relaxation oscillation frequency $(\sim 300 \mathrm{kHz})$, respectively. The schematic diagram for RIN and linewidth test is shown in Fig. 4. For RIN test, the optical fiber interferometer in the soundproof box is removed, and the laser is directly incident to the photodetector (PD). The real algorithm is according to the definition of RIN in the corresponding optical testing handbook and referenced publications [9].
RIN is the ratio of the mean-square optical intensity noise to square of the average optical power:

$$
\mathrm{RIN}=\frac{<\Delta P^{2}>}{P^{2}} \mathrm{~dB} / \mathrm{Hz} .
$$

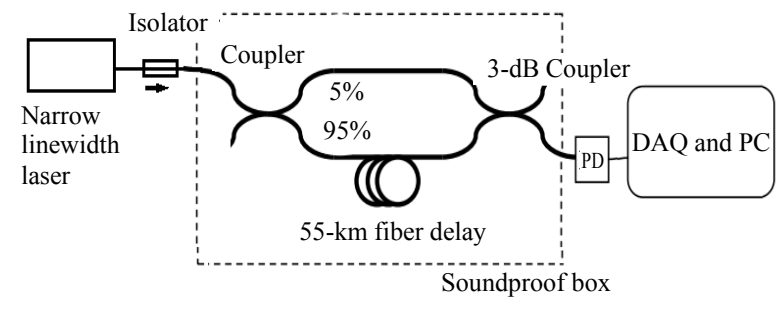

Fig. 4 Schematic diagram for RIN and linewidth test.

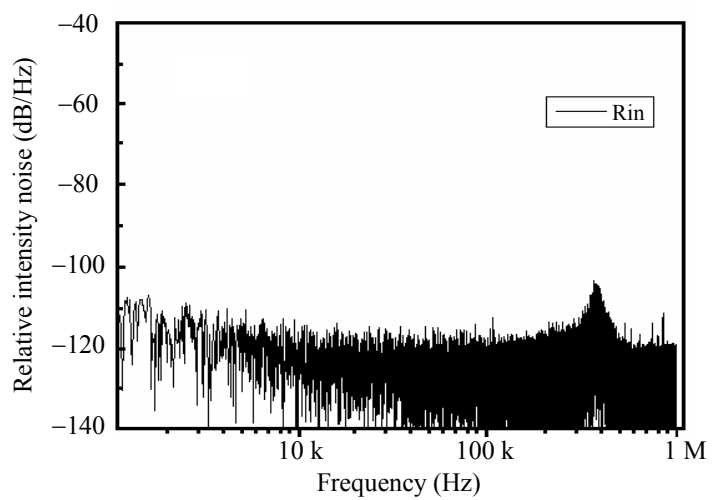

(a)

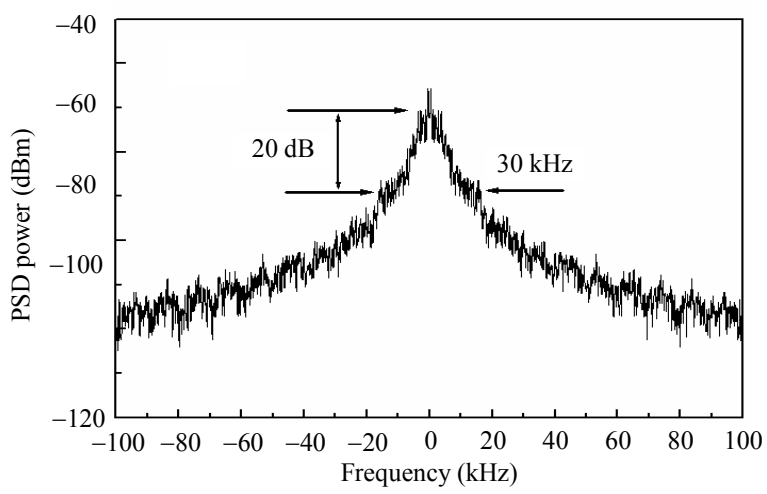

(b)

Fig. 5 Laser test results: (a) measured RIN spectra and (b) self-homodyne spectra of the filtered single wavelength narrow linewidth fiber laser.

The linewidth is measured by using the delayed self-homodyne method with a $55-\mathrm{km}$ optical fiber delay line. The beat frequency signal from an arm of the Mach-Zehnder optical fiber interferometer is detected and then processed with a personal computer (PC) to perform the power spectral density analysis. Assuming the linewidth meets the Lorentz linetype, the $20-\mathrm{dB}$ spectrum width is estimated to 
be $30 \mathrm{kHz}$, indicating the laser Lorentz linewidth is about $1.5 \mathrm{kHz}$. The tested results of RIN and linewidth are shown in Fig. 5.

\section{Results and discussion}

The main advantage of this multi-wavelength narrow linewidth fiber laser configuration is its very compact structure where there are only two pumps for eight cavities and the numbers of the corresponding optical components such as the wavelength division multiplexer (WDM) and ISO are also reduced. However, since the linewidth of the DFB-FL is very sensitive to the ambient noise, the precise temperature control for each cavity, the sound isolation packages for each cavity, and the whole laser configuration are necessary to make it stable and practical.

As for the wavelength number that this configuration can realize, it depends on the pump threshold of each laser cavity, the loss in the array, and the required power uniformity of the laser output. If the wavelength number increases, the power uniformity is more difficult to control. Usually, the pump threshold of the cavity is very low and much smaller than $5 \mathrm{~mW}$, normally $\sim 1 \mathrm{~mW}$. Hence, the laser cavity threshold is not a key problem. The laser cavity, i.e. the phase-shifted grating, is written on a short EDF, and the EDF is spliced to a piece of matched passive fiber at both ends. In the normal laser array configuration, the laser cavities with matched passive fiber sections are spliced to each other in series. Lots of splicing points make the loss in the array large in total. Hence, the real pump power in different cavities may have great differences, and lasers with different wavelengths may have large difference in power duo to the accumulated loss through different laser cavities and splicing points. If the phase-shifted gratings of different wavelengths can be written in a single line EDF without being spliced to each other, the loss in the array can be reduced largely. In the present experiment, the two pumps, P1 and P1, are properly adjusted and optimized to get the uniform power distribution.

\section{Conclusions}

In this paper, we report a multi-wavelength narrow linewidth fiber laser configuration that has a compact structure and supplies a flat power distribution. It is based on the distributed feedback fiber laser, serial cavity array, and bidirectional pump design. Currently, the eight-wavelength laser with $120-\mathrm{mW}$ total power and $1.2-\mathrm{dB}$ power difference within the wavelengths is obtained. In the next work, more wavelengths should be realized through reducing the loss in the array and adjusting the structural parameters of the laser cavities to meet the requirement in the larger scaled optical fiber sensing. The protype based on this laser configuration is just in progress in the lab.

\section{Acknowledgment}

This work is supported by the International Science and Technology Cooperation Program of China (2012DFA10730) and Natural Science Foundation of China (61307101 and 61205083).

Open Access This article is distributed under the terms of the Creative Commons Attribution 4.0 International License (http://creativecommons.org/ licenses/by/4.0/), which permits unrestricted use, distribution, and reproduction in any medium, provided you give appropriate credit to the original author(s) and the source, provide a link to the Creative Commons license, and indicate if changes were made.

\section{References}

[1] G. A. Cranch, G. M. H. Flockhart, and C. K. Kirkendall, "Comparative analysis of the DFB fiber laser and fiber-optic interferometric strain sensors," SPIE, 2007, 6619: 66192C.

[2] C. Wang, C. Wang, Y. Shang, X. Liu, and G. Peng, "Distributed acoustic mapping based on interferometry of phase optical time-domain reflectometry," Optics Communications, 2015, 346: 172-177.

[3] G. A. Cranch, C. K. Kirkendall, K. Daley, S. Motley, A. Bautista, J. Salzano, et al., "Large-scale remotely 
pumped and interrogated fiber-optic interferometric sensor array," IEEE Photonics Technology Letter, 2003, 15(11): 1579-1581.

[4] J. T. Kringlebotn, J. L. Archambault, L. Reekie, and D. N. Payne, "Er3+:Yb3+-codoped fiber distributed feedback laser," Optics Letter, 1994, 19(24): 2102-2103.

[5] Q. Li, F. Yan, W. Peng, T. Feng, S. Feng, S. Tan, et al., "DFB laser based on single mode large effective area heavy concentration EDF," Optics Express, 2012, 20(21): 23684-23689.

[6] H. Qi, Z. Song, S. Li, C. Wang, and G. Peng, "Short distributed feedback fiber laser with unidirectional output for sensing applications," Chinese Optics Letter, 2013, 11(4): 44-46.

[7] H. Qi, Z. Song, J. Guo, C. Wang, and G. Peng,
"Apodized distributed feedback fiber laser with asymmetrical outputs for multiplexed sensing applications," Optics Express, 2013, 21(9), 11309-11314.

[8] S. Foster, A. Tikhomirov, and M. Englund, "A 16 channel fibre laser sensor array," in 18th Proc. ACOFT/AOS, Melbourne, Australia, pp. 40-42, 2006.

[9] Y. Léguillon, K. H. Tow, P. Besnard, A. Mugnier, D. Pureur, and M. Doisy, "First demonstration of a 12 DFB fiber laser array on a $100 \mathrm{GHz}$ ITU grid, for underwater acoustic sensing application," SPIE, 2012, 8439: 84390J.

[10] H. Qi, Z. Song, J. Guo, J. Ni, C. Wang, and G. Peng, "Narrow-linewidth distributed feedback fiber laser with MOPA," Chinese Optics Letter, 2015, 13(S2): S21404. 